\title{
Only irrelevant angry, but not happy, expressions facilitate the response inhibition
}

\author{
Rashmi Gupta $^{1}$ - Jay Prakash Singh ${ }^{1}$ \\ Accepted: 21 October 2020 / Published online: 4 November 2020 \\ (C) The Psychonomic Society, Inc. 2020
}

\begin{abstract}
It has been debated that arousal rather than valence modulates the response-inhibition process. The processing of irrelevant information of happy and angry faces interacts with attention differently. In the present study, arousal-matched irrelevant happy and angry faces were used as stop-signals in the stop-signal paradigm. Participants were required to respond to go-signals (discriminate between $\mathrm{X}$ or $\mathrm{O}$ ). Occasionally, a stop-signal was presented where participants were required to withhold their motor response. Results indicate a significant effect of emotion on response inhibition, which suggests that valence of a stopsignal modulates inhibitory control. More specifically, we found that only irrelevant angry, but not happy, expressions facilitate the response inhibition process. These results have theoretical implications for understanding the nature of emotions and its interaction with cognitive control functions.
\end{abstract}

Keywords Emotions $\cdot$ Attention $\cdot$ Response inhibition $\cdot$ Happy face $\cdot$ Angry face

\section{Introduction}

Emotions play an essential role in our life. The processing of emotional stimuli is vital for survival because they provide crucial social and environmental information/cues. When many stimuli in the environment compete for processing resources, emotional stimuli receive prioritization over neutral stimuli and therefore affect cognitive processes (Kalanthroff et al., 2013). Maladaptive processing of emotional information can lead to various psychopathological conditions (e.g., depression, anxiety, post-traumatic stress disorder). Therefore, understanding the nature of the interaction between cognition processes and emotions is essential (Cisler et al., 2010; Gupta, 2007; Gupta \& Kar, 2008, 2012; Sippel \& Marshall, 2013).

\section{Attention and emotion (positive and negative)}

Ample research indicates that both "emotional states" and "emotional stimuli" modulate attention processes (see Gupta, 2019; Pessoa, 2009; Vuilleumier, 2005, for a

Rashmi Gupta rash_cogsci@yahoo.com

1 Cognitive and Behavioural Neuroscience Laboratory, Department of Humanities and Social Sciences, First Floor, Indian Institute of Technology Bombay, Mumbai, Maharashtra 400076, India review). For example, positive emotional states (e.g., joy, contempt) and positive stimuli (e.g., happy faces, reward) broaden and distribute the scope of attention. In contrast, negative states (e.g., depression, anxiety) and negative stimuli (e.g., sad and angry faces) narrow or focus the scope of attention (Fredrickson, 2004; Gupta, 2019; Gupta \& Srinivasan, 2015; Gupta et al., 2016; Srinivasan \& Gupta, 2010, 2011). These studies indicate that positive and negative emotions interact with attention differently.

Emotional stimuli enhance (when used as a target) and interfere (when used as a distractor) with various cognitive processes. For example, emotional stimuli, especially negative emotional stimuli, capture attention compared to positive and neutral stimuli (Eastwood, Smilek, \& Merikle, 2001; Frischen, Eastwood, \& Smilek, 2008). Shallower slopes of visual search functions reflect higher guidance on the focus of attention, which indicates that angry faces guide focal attention more effectively than happy faces (Fox et al., 2000). However, the preferential detection of happy faces was observed over angry faces in some studies (Becker et al., 2011; Savage et al., 2016). Moreover, when less attentional resources are available, happy faces capture attention compared to angry faces because fewer attention resources are required to process it (Gupta, 2019, for a review; Gupta et al., 2016; Gupta \& Srinivasan, 2015; Gupta, 2016; Gupta et al., 2019; Gupta \& Raymond, 2012; Leppänen \& Hietanen, 2004; Mack \& Rock 1998; Pandey \& Gupta, 2019). 
In line with this view, Gupta et al. (2016) conducted a series of experiments where positive (happy faces, erotic images, and neutral faces associated with the gain outcome) and negative (angry faces, mutilated bodies, and neutral faces associated with loss outcome) images were used as distractors in a primary letter-search task (low and high load). Participants were required to ignore distractor images and detect a target letter (X or $\mathrm{N}$ ) among five circular letters (all Os: low-load condition) or five angular letters (Z, W, H, K, and M: highload condition). The authors found that both positive and negative distractors captured the attention and interfered with the letter-search performance in the low-load task. However, when attention was constrained (high-load condition), only positive (not negative) distractors interfered with the primary letter search task. Consistent with this finding, the higher recognition accuracy of happy faces (relative to sad faces) under high-load conditions in an inattentional blindness task was reported (Gupta \& Srinivasan 2015; Mack \& Rock, 1998).

The prioritization of positive stimuli over negative stimuli was also found in the temporal selection paradigm. For example, using rapid serial visual presentation (RSVP) task, Most et al. (2007) presented a series of neutral images where one image (positive or negative) was used as a distractor that was followed by a neutral target image. Only the target image was rotated $90^{\circ}$ to the left or right. Participants were required to detect the orientation of the target image while ignoring the distractor. They found that positive distractors involuntarily oriented attention and interfered with identifying the target more than negative distractors did.

\section{Response inhibition and emotion (positive and negative)}

It is essential to ignore/inhibit irrelevant emotional information to perform well on a primary task (Kalanthroff et al., 2013; Verbruggen et al., 2004). Response inhibition has been defined as "the suppression of inappropriate actions in a given context, and that interfere with goal-driven behavior" (Mostofsky \& Simmonds, 2008, p. 751). Attention plays an essential role in inhibiting irrelevant information (Lavie, 2005, for a review). Both "emotion" (Kalanthroff et al., 2013; Pessoa et al., 2012; Verbruggen \& de Houwer, 2007) and "attention" (Scalzo et al., 2016; Tian et al., 2014) modulate response inhibition processes. Given a link between attention, emotion, and response inhibition, the present study examines the role of irrelevant positive and negative emotions in response inhibition/inhibitory control.

A handful of studies have explored the link between irrelevant emotional information and response inhibition using a stop-signal paradigm, but the results are inconsistent and inconclusive (Kalanthroff et al., 2013; Pawliczek et al., 2013; Pessoa et al., 2012; Sagaspe et al., 2011; Verbruggen \& de Houwer, 2007). For example, using a modified version of the stop-signal task, Kalanthroff et al. (2013) presented negative or neutral images as a prime that was followed by a visual gosignal (@ or \#) to which participants were required to press the corresponding keys. Occasionally, an auditory stop-signal was presented on which participants were required to withhold their motor response. Results indicated that the presentation of negative primes (relative to neutral) increased both response and stopping latencies. In another study, faces with irrelevant fearful and neutral expressions were used as gosignals, and participants were required to detect the gender (male vs. female) (Sagaspe et al., 2011). The authors found that irrelevant, fearful information prolonged response latencies but not stopping latencies. Fearful information also did not modulate the neural circuits involved in response inhibition processes.

Notably, in most of the studies, only negative emotion was incorporated and was compared against neutral stimuli. Negative and neutral stimuli not only differ on valence but also on the arousal level. For example, negative emotional stimuli are more arousing compared to neutral stimuli (Lohani et al., 2013). Therefore, prolonged stopping latencies could be due to the arousal difference between negative and neutral information (see Pessoa et al., 2012, Experiment 1 for similar effect and interpretation).

The role of positive emotion in response inhibition is unclear. To get a complete picture of emotion-based modulation in response inhibition, it is crucial to compare positive and negative emotions directly (Gupta, 2019; Pandey \& Gupta, 2019) while controlling their arousal difference. There is only one study where authors have manipulated the valence (positive vs. negative) and the arousal value (high vs. low) of the stimuli orthogonally in the stop-signal task. They found that irrelevant, high-arousing positive and negative emotional stimuli (relative to low arousing positive and negative stimuli) impaired the stopping latencies. In this study, irrelevant emotional stimuli were not part of the stop-signals; instead, they were presented as primes before the go and stop-trials. Also, in this study, IAPS pictures were used as stop-signals, whereas in previous (Pessoa et al., 2012) and the current study, faces were used as stop-signals. Notably, IAPS pictures are quite complex. Also, low-level visual features varied a lot between positive and negative pictures (e.g., negative images such as mutilated bodies are dominated by red color, whereas the skin color dominates positive images such as erotic pictures). Therefore, processing of these complex images could have taken more resources, thereby interfering with the response inhibition in Verbruggen and de Houwer's (2007) study.

There is another study where both positive and negative stimuli were used as stop-signals (Pessoa et al., 2012, Experiment 1). However, the arousal level of happy, fearful, and neutral faces was not measured and controlled. They used low-threat stimuli (happy or fearful faces in Experiment 1) and high-threat stimuli (digits paired with shock in Experiment 2) 
as stop-signals. They found that a low-threat stop-signal improved response inhibition, whereas a high-threat stop-signal impaired response inhibition. Authors have speculated that both positive and fearful faces are low in arousal level, and therefore, generated enhanced sensory representations of the stop-signal in the visual cortex (Pessoa et al., 2002; Vuilleumier, 2005), which in turn facilitated response inhibition in Experiment 1. In contrast, stop-signals impaired response inhibition in Experiment 2. Authors argued that in Experiment 2, negative stop-signals (words associated with shock) were threatening and high arousing that might have consumed most of the central processing resources required for inhibition (as suggested by the "dual competition framework"; Pessoa, 2009). Notably, in Experiment 2, stop-signals with positive emotion were not included in their study. Therefore, it is unclear whether stop-signals with a positive emotion would modulate the response inhibition. Moreover, without measuring and controlling the arousal level of stopsignals, Pessoa et al. (2012) concluded that the arousal but not valence of stop-signals modulates response inhibition. Therefore, to what extent is this conclusion justifiable?

Notably, Pessoa et al. (2012) compared happy faces with fearful faces and did not find the difference. This could be attributed to the motivational tendencies (approach/avoidance) modulated by these faces as the perception of both faces activates approach-related behavior (Hammer \& Marsh, 2015; Fusar-Poli et al., 2009) and a similar neural response (FischerShofty et al., 2010; Marsh et al., 2010). In addition, these faces do not signal danger and can easily be approached (Nikitin \& Freund, 2019; Grose-Fifer et al., 2013). Neuroimaging studies further strengthen this claim. For example, the administration of oxytocin in healthy adults increases approach-related behavior sensitivity to both fearful and happy facial expressions (Fischer-Shofty et al., 2010; Marsh et al., 2010). A quantitative voxel-based meta-analysis of the brain has shown that happy and fearful faces specifically activated the amygdala brain region. Therefore, to what extent is the comparison between happy faces and fearful faces meaningful?

Given these similar motivational and neural responses evoked by happy and fearful faces, we argue that it is better to compare happy and angry faces against each other in the stop-signal paradigm because they each produce a specific and different motivational and neural response. For example, the perception of happy and angry faces strongly activates approach and avoidance related behaviors, respectively (Marsh et al., 2005; Nikitin \& Freund, 2019). Neuroimaging studies further strengthen this claim. The amygdala was activated in response to happy faces, whereas the insula was activated in response to angry faces (Fusar-Poli et al., 2009). We also discussed above that angry and happy faces interact with attention differently (see Gupta, 2019, for a review; Gupta et al., 2016; Mack \& Rock 1998; Srinivasan \& Gupta, 2011; Gupta \& Srinivasan, 2015; Gupta \& Deák, 2015).
There is only one study where angry emotional faces were compared against neutral faces in the stop-signal paradigm (Pawliczek et al., 2013). The authors found that angry faces facilitated response inhibition compared to neutral faces. Similar to previous studies, this study also did not control the arousal level of emotional faces; the arousal level of angry faces could be more in comparison to neutral faces. Notably, happy faces were not used in this study. Therefore, this study was also unable to comment on the effect of valence (positive vs. negative) on response inhibition.

\section{The present study}

Given a difference in the interaction between positive and negative emotion with attention, as well as the role of attention in response inhibition, the present study aimed to examine the role of valence (happy vs. angry) in response inhibition. Notably, happy and angry faces used in the present study were matched on the arousal level. Also, we aimed to better understand the role of valence rather than low-level visual differences between emotional faces that are visually similar but vary in emotional expressions (happy vs. angry). These are the strengths of the present study.

Based on previous findings, two contrary hypotheses are formulated. "Dual competition framework" (Pessoa, 2009) predicted that happy faces would facilitate the response inhibition (smaller SSRT value) because it requires fewer resources to process and leaving enough resources required for response inhibition. In contrast, an angry stopsignal would interfere with the response inhibition (higher SSRT value) because it consumes more resources to process and leaves fewer resources for response inhibition. In contrast to the prediction of the "Dual competition framework," "Approach and avoidance framework" (Hammer \& Marsh, 2015; Marsh et al., 2005) predicted that happy stop-signal would reduce the response inhibition (higher SSRT value) because it facilitates approach-related behavior. Also, happy faces are appetitive in nature. In contrast, angry stop-signal would facilitate response inhibition (smaller SSRT value) because it facilitates avoidancerelated behavior and is perceived as aversive. In line with this framework, it has been reported that "it is difficult to disengage attention from an angry face once it is seen" (Becker et al., 2019, p. 107). Authors found that angry faces hold the attention and resist attentional disengagement more than happy faces. This may suggest that angry faces produce freezing behavior, and therefore, are more likely to facilitate response inhibition than happy faces are.

Since the arousal level of both happy and angry faces was matched in the present study, therefore, the difference between happy and angry faces in response inhibition would be completely attributed to valence difference. 


\section{Methods}

\section{Participants}

Sixty young adults (43 males, $M_{\text {age }}=26.48$ years, $S D=5.58$ years) participated after giving informed consent. We estimated (using G-Power) a necessary sample size of 45 to detect a medium-size effect of 0.25 (Cohen, 1988), and to obtain a power level of 0.95. All participants were right-handed with normal or corrected-to-normal vision and with no past neuropsychiatric disorder. The ethics committee of the Indian Institute of Technology Bombay approved the study.

\section{Apparatus}

Stimuli were displayed on a 15.6-in. color monitor $(60 \mathrm{~Hz}$; resolution $1,920 \times 1,080$ pixels) viewed binocularly from a distance of $60 \mathrm{~cm}$. Octave (an open-source version of the MATLAB interpreter) (Brainard, 1997; Kleiner et al., 2007) operating on a computer with a $2.0 \mathrm{GHz}$ Ryzen 5 processor generated stimuli and recorded responses acquired via a number pad.

\section{Stimuli}

A total of four static grey-scale photographs of young adults (two male and two female identities with three emotions) were selected as stop-signals from the Karolinska Directed Emotional Faces (KDEF) (Lundqvist, Flykt, \& Öhman, 1998), and the Nimstim Face Stimulus Set (Tottenham et al., 2009). Each face displayed all three emotions (happy, angry, and neutral) and subtended approximately $2.1^{\circ} \times 2.9^{\circ} .31(17$ males, $M_{\text {age }}=28.8$ years, $S D=4.17$ years) healthy participants, who did not take part in the main experiment, rated these original faces on arousal level. Each emotion of a face was presented at a time on a computer screen during the rating phase. Participants were required to make a response by selecting one of the three emotion options of emotion: happy, angry, and neutral. After this, participants had to choose a number that corresponded with the arousal level of a target face from a Likert scale ranging from 1 to 9, where one indicated "not at all aroused" and nine indicated "very aroused."

A two-way ANOVA was performed on arousal score using gender of the faces (male vs. female) and emotion (happy, angry, neutral) as within-group variables. The main effect of emotion was significant, $F(2,60)=15.57, M S E=5.52, p<$ $.0001, \eta_{p}{ }^{2}=.342$. The arousal score was significantly lower for the neutral faces $(M=4.37, S D=2.26)$ compared to angry $(M=6.60, S D=1.72), t(1,30)=4.19, p<.0001$, and happy faces $(M=6.13, S D=1.55), t(1,30)=4.38, p<.0001$. There was no significant difference in the arousal score between angry and happy faces, $t(1,30)=1.57, p=.126$. There was no significant main effect of gender of the faces, $F(1,30)=$ $0.580, M S E=1.56, p=.452, \eta_{p}{ }^{2}=.019$, which indicates that arousal score was similar for male $(M=5.63, S D=1.32)$ and female faces $(M=5.77, S D=1.45)$. There was no significant interaction between face gender and emotion on arousal ratings, $F(2,60)=0.491, M S E=1.43, p=.615, \eta_{p}{ }^{2}=.016$.

\section{Procedure for the stop-signal task}

The stop-signal paradigm (Gupta et al., 2011; Gupta \& Kar, 2009) was used to measure response inhibition (see Fig. 1). There were two types of trials: go and stop-trials. Each trial starts with a fixation cross $\left(0.49 \times 0.49^{\circ}\right)$ at the center of the screen for $500 \mathrm{~ms}$, which was followed by the presentation of a letter " $\mathrm{X}$ " $\left(2.2^{\circ} \times 2.4^{\circ}\right)$ or " $\mathrm{O}$ " $\left(2.3^{\circ} \times 2.5^{\circ}\right)$ for $1,000 \mathrm{~ms}$. In go-trials subjects were required to indicate " $\mathrm{X}$ " or " $\mathrm{O}$ " via key-press, using their right-hand, which stayed on the screen for 1,000 ms irrespective of participants' response and was followed by a blank screen for 2,000 ms. Stop-trials were similar to the go-trials except that a brief picture of a face (happy, angry, or neutral) was presented above the letter " $\mathrm{X}$ " or "O" for $250 \mathrm{~ms}$ after a random duration with a variable stop-signal delay (SSD) relative to the onset of the go stimulus. Each face was separated from the letter " $\mathrm{X}$ " or " $\mathrm{O}$ " by $0.20^{\circ}$. The initial value of the SSD was set at $250 \mathrm{~ms}$ for all three stop conditions and adjusted by a staircase tracking procedure. After each successful stopping the SSD was increased by $50 \mathrm{~ms}$ on a subsequent stop trial and after each unsuccessful stopping the SSD was reduced by $50 \mathrm{~ms}$ on a subsequent stop trial (Logan et al., 1997; Pessoa et al., 2012). This staircase was run separately for each condition to ensure successful inhibition of approximately $50 \%$ of the stop trials in each condition. Participants were required to respond to letters as quickly and as accurately as possible and withhold their motor response upon viewing a face that followed the letters. Equal emphasis was given on executing and withholding the motor response. A full practice run was given before the experimental session. In the practice run, a separate set of neutral faces was used as a stop-signal.

There were a total of 192 trials in the experiment. A total of four blocks was completed. Each block contained a total of 48 trials (36 go-trials (75\%) and 12 stop-trials (25\%; four for each of the three-stop conditions). Each stop-trial was followed by at least one go-trial. " $\mathrm{X}$ " and "O" appeared for the equal number of times in both go and stop trials.

\section{Data analysis}

Data of ten participants were removed from the analysis due to excessive omission errors (inability to respond to go-stimulus on go-trials) rate $(\geq 13 \%$; omission error rate range $=13-6 \%$; mean omission error rate $=21.87 \%, \mathrm{SD}=14.24 \%$ ), which indicates that these participants were waiting for the stopsignals for a more extended period and missed to respond on go-trials. Also, the data of five participants were removed 
Go Trials

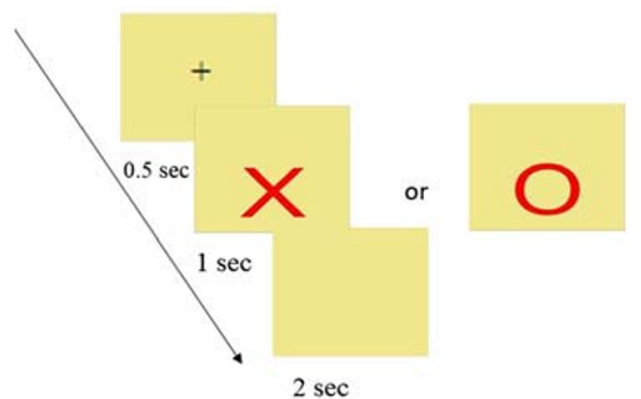

Fig. 1 An example of go and stop-trials. During go trials, participants responded to go-signals ( $\mathrm{X}$ or $\mathrm{N}$ ?). During stop-trials, they were required to withhold their motor response (signaled by a face with irrelevant happy, angry, or neutral emotions). The stop-signals followed the go-signals

from the analysis due to excessive commission error (inability to withhold motor response on stop-trials), rate ( $\geq 75 \%$, range $=89-100 \%$ ), which indicates that these participants were not able to pay attention in the task (see footnote ${ }^{1}$, for detailed discussion).

In the final analysis, there were 45 participants (33 males, $M_{\text {age }}=26.02$ years, $S D=5.37$ years; mean omission error rate $=3.47 \%, S D=3.54 \%$ ). Stop-signal reaction time (SSRT), which is a measure of response inhibition time, was calculated $\left(\right.$ see footnote ${ }^{2}$ ) for each participant in each condition as median RT during correct go-trials minus median SSD (SSD values: happy: $M=268.88, \mathrm{~ms}, S D=135.38 \mathrm{~ms}$; angry: $M$ $=291.66 \mathrm{~ms}, S D=133.56 \mathrm{~ms}$; neutral: $M=280.55, S D=$ $136.08 \mathrm{~ms}$ ) (Logan \& Cowan, 1984; Verbruggen \& Logan, 2009). A one-way repeated-measure ANOVA was performed on SRRT values using stop-signal type (happy, angry, and neutral) as a within-group factor. The SSD was adjusted using the stair-casing method for each stop-signal type separately to achieve an approximately $50 \%$ success rate of inhibition. Likewise, one-way repeated-measure ANOVAs was

\footnotetext{
${ }^{1}$ Previous studies have reported the omission error rate in the range of $1-6.5 \%$ in the stop-signal task (Pessoa et al., 2012; Verbruggen \& de Houwer, 2007). It has also been shown that participants make a lot of errors in the primary task in the presence of irrelevant emotional information such as happy and angry faces (range 10-35\%) (Gupta et al., 2016). Therefore, we doubled the no. of omission rates $(\geq 13 \%)$ to exclude participants from the final data set. Based on these criteria, data of 10 participants was removed (omission error rate range $13-56 \%$; mean omission error rate $=21.87 \%, \mathrm{SD}=14.24 \%$ ), indicating that these participants were waiting for the stop-signals for a more extended period and missed to respond on go-trials. Also, data of five participants were removed from the analysis due to excessive rate $(\geq 75 \%$, range $=89-100 \%)$ of commission error, which indicates that these participants were not able to pay attention to the task mostly.

${ }^{2}$ Notably, in the present study median value (which is insensitive to extreme value) of Go-RT and median SSD was used to calculate SSRT; therefore, it rules out the potential concern of small no. of trials with extreme values.
}

Stop rials

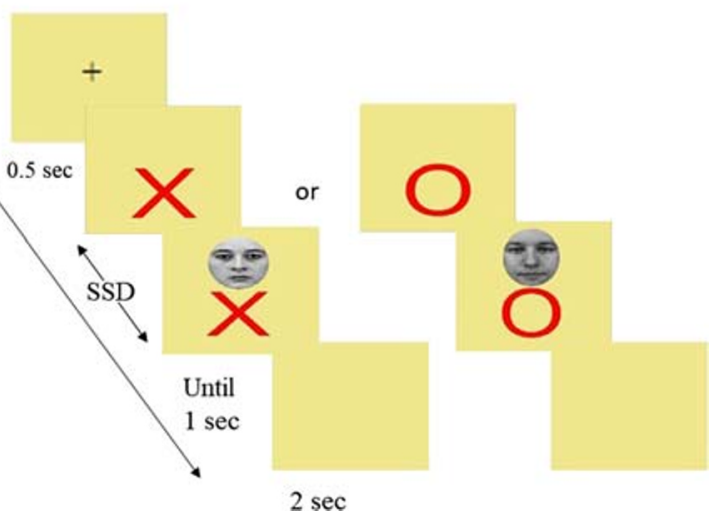

after a variable delay called stop-signal delay. The SSD was updated based on a staircase procedure separately for each stop-signal condition to get stop-performance at approximately $50 \%$ correct. SSD Stop-Signal Delay

performed on commission error and stop-respond RT (unsuccessful stop-trials (UNSUCC): stop trials on which participants failed to inhibit the motor response) (Pessoa et al., 2012). Alpha level was set to 0.05 .

\section{Results}

The one-way ANOVA on SRRT score yields a significant main effect of stop-signal type, $F(2,88)=3.52, M S E=$ $1657.09, p=0.034, \eta_{p}{ }^{2}=.074$, which suggests that irrelevant emotional information modulates response inhibition (see Fig. $2)$. The SSRT score was significantly lower for angry $(M=$ $307.6 \mathrm{~ms}, S D=50.52 \mathrm{~ms})$ compared to happy $(M=330.4, \mathrm{~ms}$, $S D=61.3 \mathrm{~ms}), t(44)=-2.35, p=0.02$, but close to significantly different for neutral $(M=318.8, S D=52.4 \mathrm{~ms}), t(44)=$ $1.86, p=0.070$, stop-signal. This may indicate that an angry stop-signal facilitated the response inhibition. There was no significant difference in SSRT between the happy and neutral conditions, $t(44)=-1.22, p=.22$., which may indicate that both happy and neutral stop-signal modulate response inhibition similarly. As expected, the one-way ANOVA on commission error rate yields a non-significant main effect of stopsignal type, $F(2,88)=1.26, M S E=19.27, p=0.28, \eta_{p}{ }^{2}=$ .268 , (neutral: $M=41.52 \%, S D=13.98 \%$; happy: $M=$ $42.63 \%, S D=13.34 \%$; angry: $M=41.25 \%, S D=13.41 \%$ ), which indicates that stop performance was approximately $50 \%$ correct during all three emotional conditions. Finally, there was no significant main effect of a stop-signal type on stop-respond RT, $F(2,88)=.402, M S E=2999.42, p=.67, \eta_{p}{ }^{2}$ $=.009$. The reaction times of stop-respond (UNSUCC) trials were significantly faster than those of correct go trials (angry: $t(44)=6.80, p=.001$; happy: $t(44)=8.09, p=.001$; neutral: $t(44)=5.97, p=.001)$, as predicted by the race model (Logan \& Cowan, 1984). 
350.0

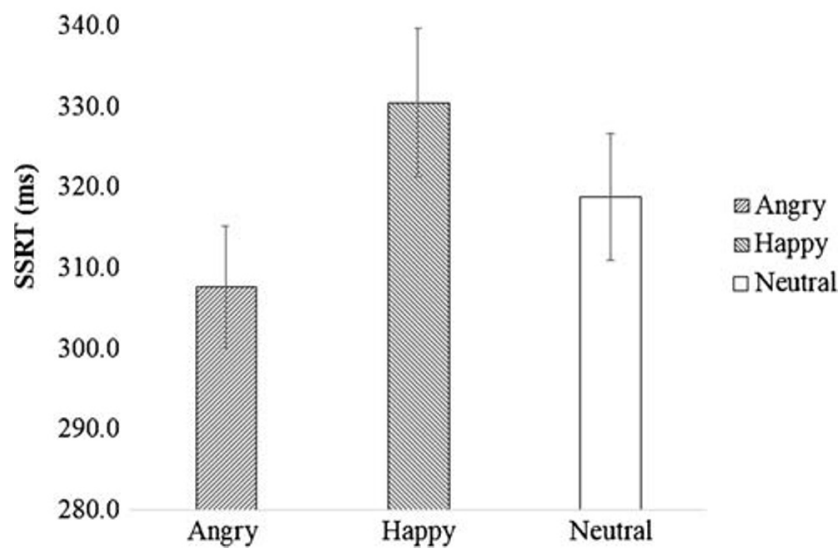

Fig. 2 Mean SSRT was plotted as a function of emotion. Vertical lines represent \pm 1 within-subject standard error. SSRT Stop-Signal Reaction Time

\section{Discussion}

The present study is the first study where faces with irrelevant positive (happy) and negative (angry) expressions were used as stop-signals and compared with the neutral faces to examine the response inhibition process. Moreover, both happy and angry faces were matched on the arousal level. The SSRT value was smaller for angry face stop-signals than for happy and neutral face stop-signals; therefore, we argue that the valence (positive and negative) of the stop-signals matter and that they modulate response inhibition.

The present study holds many similarities to Pessoa et al.'s (2012) study (see footnote ${ }^{3}$ ). However, the results are quite different. Pessoa et al. (2012) found a facilitatory effect of the irrelevant emotional information on response inhibition, irrespective of the positive and negative valence of the stopsignals (see Experiment 1). However, in the present study, the facilitatory effect of irrelevant emotional information was only observed for angry faces compared to happy and neutral faces. This result is consistent with the prediction of "approach and avoidance framework," but inconsistent with the prediction of "dual competition framework." Why only irrelevant angry face stop-signals, but not happy face stop-signals, facilitated response inhibition in the present study? There could be several explanations, which are discussed next.

\footnotetext{
$\overline{3}$ For example, in both studies, faces with irrelevant positive (happy faces) and negative emotion (fearful faces in their study and angry in the present study) were used as the stop-signals. Also, non-emotional and non-face stimuli were used as go-signals in both studies ("circle" or "square" in their study; " $\mathrm{X}$ " or "N" in the present study). However, in their study, both the go and the stopsignals were presented at the foveal vision (center), whereas in our study, gosignals were presented at the center, and stop-signals were presented at the top of the go-signals (Gupta et al., 2011). The arousal level of positive and negative emotional stimuli used in Pessoa et al.'s (2012) study was neither measured nor controlled, which is the major drawback of their study. However, one of the merits of the present study is that the arousal level of positive and negative emotion was measured and controlled.
}

First, it has been observed that angry emotion elicits avoidance reactions if contrasted with the expression of happiness. However, when angry emotion was contrasted with other negative emotions like fear and sadness, it produces an approach tendency (Paulus \& Wentura, 2016). In the present study, angry faces were contrasted with happy faces, whicht may have activated the avoidance response and defensive motivational system that facilitates freezing behavior ("a statue-like inhibition of movement" (Facchinetti et al., 2006; Lang \& Bradley, 2013; Lang et al., 2000) and may have held attention (Becker et al., 2019), which in turn facilitated response inhibition. These explanations are consistent with the prediction of the "Approach and avoidance framework." In the present study, no other negative emotional faces (e.g., fearful, sadness) were included; therefore, we were not able to examine the differential effect of different negative emotions on response inhibition. Future studies should include arousal matched different negative emotional faces (e.g., angry, fearful, sad) in the same study to examine this.

Second, it has been observed that when fewer attentional resources are available, happy faces capture attention and interfere with the primary task more compared to angry faces (Gupta, 2019, for a review; Gupta et al., 2016). In the present study, we found the same results that irrelevant happy emotion of the stop-signals reduced response inhibition (primary task) more compared to angry face stop-signals.

Third, the behavioral results of the present study may indicate that the processing of irrelevant angry faces may have modulated neural processing pathways involved in response inhibition (Pawliczek et al., 2013). Neuroimaging studies indicated that the stop-signal on angry face trials activated the occipital brain areas compared to the neutral face. It may reflect higher representation or higher attention processing of angry faces, leading to a facilitation effect in response inhibition compared to happy and neutral faces (Pawliczek et al., 2013). In line with this view, it has been suggested that the detection of angry faces is fast and efficient (Fox et al., 2000), which may indicate an enhanced representation of angry faces in the visual cortex compared to happy and neutral faces. Also, Pawliczek et al. (2013) have found increased activation in the bilateral fusiform gyrus (see also Jackson et al., 2008; Kesler-West et al., 2001) and posterior cingulate cortex (PCC) (see also Sprengelmeyer et al., 1998) in response to angry faces. Activation in both brain areas has also been found for emotional and non-emotional inhibition tasks. Higher activation in the PCC supports that major attentional resources were allocated to process angry faces (Boehler et al., 2009). Together, higher activation in all three brain areas (visual cortex, fusiform gyrus, and PCC) in response to angry face stimuli may have facilitated response inhibition (Pawliczek et al., 2013). The present study should be replicated with neuroimaging studies to test these explanations.

To summarize, the results of the present study indicate that stop-signals with irrelevant angry facial expressions facilitate 
response inhibition compared to stop-signals with irrelevant happy and neutral facial expressions. Our results extend previous findings by suggesting the valence of the stop-signals modulates inhibitory control. We propose comparing happy faces against angry faces to examine the role of valence (positive and negative) on response inhibition. The "approach and avoidance framework" and "freezing account" of emotion can explain the results of the present study compared to the "dual competition framework." Therefore, it is essential to test the "dual competition framework" using other facial expressions. Future studies should include arousal matched angry, happy, and fearful faces as stop-signals in a paradigm used in Pessoa et al.'s (2012) study to strengthen the results of the present study, which will further help examine the role of valence in response inhibition.

Funding This research was supported by the IRCC, IITB seed grant (RD/ 0518-IRCCSH0-013) to Prof. Gupta.

\section{Compliance with ethical standards}

Conflict of interest All authors declare no conflicts of interest.

Ethical approval All procedures performed in studies involving human participants were in accordance with the ethical standards of the institutional and/or national research committee and with the 1964 Helsinki declaration and its later amendments or comparable ethical standards.

Informed consent Informed consent was obtained from all individual participants included in the study.

\section{References}

Becker, D., Rheem, H., Pick, C. M., Ko, A., \& Lafko, S. R. (2019). Angry faces hold attention: Evidence of attentional adhesion in two paradigms. Progress in Brain Research, 247, 89-110.

Becker, D. V., Anderson, U. S., Mortensen, C. R., Neufeld S. L., \& Neel, R. (2011). The Face in the Crowd Effect Unconfounded: Happy Faces, Not Angry Faces, Are More Efficiently Detected in Singleand Multiple-Target Visual Search Tasks. Journal of Experimental Psychology: General, 140, 637-59

Boehler, C., Münte, T., Krebs, R., Heinze, H., Schoenfeld, M., \& Hopf, J. (2009). Sensory MEG responses predict successful and failed inhibition in a stop-signal task. Cerebral Cortex, 19, 134-145.

Brainard, D. H. (1997). The Psychophysics Toolbox. Spatial Vision, 10(4), 433-436.

Cisler, J. M., Olatunji, B. O., Feldner, M. T., \& Forsyth, J. P. (2010). Emotion Regulation and the Anxiety Disorders: An Integrative Review. Journal of Psychopathology ans Behavioral Assessment, 32(1), 68-82.

Cohen, J. (1988). Statistical power analysis for the behavioral sciences (2nd ed.). Hillsdale, NJ: Lawrence Erlbaum Associates.

Eastwood, J. D., Smilek, D., \& Merikle, P. M. (2001). Differential attentional guidance by unattended faces expressing positive and negative emotion. Perception \& Psychophysics, 63, 1004-1013.

Facchinetti, L.D., Imbiriba, L.A., Azevedo, T.M., Vargas, C.D., \& Volchan, E. (2006). Postural modulation induced by pictures depicting prosocial or dangerous contexts. Neuroscience Letters, 410, 52-56.

Fischer-Shofty M., Shamay-Tsoory S. G., Harari H., \& Levkovitz Y. (2010). The effect of intranasal administration of oxytocin on fear recognition. Neuropsychologia, 48, 179-184.

Fox, E., Lester, V., Russo, R., Bowles, R. J., Pichler, A., \& Dutton, K. (2000). Facial Expressions of Emotion: Are Angry Faces Detected More Efficiently?. Cognition \& Emotion, 14(1), 61-92.

Fredrickson B. L. (2004). The broaden-and-build theory of positive emotions. Philosophical Transactions of the Royal Society of London. Series B, Biological sciences, 359(1449), 1367-1378.

Frischen, A., Eastwood, J. D., \& Smilek, D. (2008). Visual search for faces with emotional expressions. Psychological Bulletin, 134(5), 662-676.

Fusar-Poli, P., Placentino, A., Carletti, F., Landi, P., Allen, P., Surguladze, S., Benedetti, F., Abbamonte, M., Gasparotti, R., Barale, F., Perez, J., McGuire, P., \& Politi, P. (2009). Functional atlas of emotional faces processing: a voxel-based meta-analysis of 105 functional magnetic resonance imaging studies. Journal of psychiatry and Neuroscience, 34(6), 418-432.

Grose-Fifer, J., Rodrigues, A., Hoover, S., \& Zottoli, T. (2013). Attentional capture by emotional faces in adolescence. Advances in Cognitive Psychology, 9, 81-91.

Gupta, R. (2007). Metacognitive Rehabilitation of Autobiographical Overgeneral Memory. The Journal of Cognitive Rehabilitation, 25(2), 4-8.

Gupta, R. (2016). Commentary: Neural control of vascular reactions: Impact of emotion and attention. Frontiers in Psychology, 7, 1613.

Gupta, R. (2019). Positive emotions have unique capacity to capture attention. Progress in Brain Research. 16, 144-149.

Gupta, R., \& Deák, G.O. (2015). Disarming smiles: irrelevant happy faces slow post-error responses. Cognitive Processing, 16(4), 427434.

Gupta, R., Hur, Y., \& Lavie, N. (2016). Distracted by Pleasure: Effects of Positive Versus Negative Valence on Emotional Capture Under Load. Emotion, 16, 328-337.

Gupta, R., \& Kar, B. R. (2008). Interpretative Bias: Indicators of Cognitive Vulnerability to Depression. German Journal of Psychiatry, 11, 98-102.

Gupta, R. \& Kar, B. R (2009). Development of attentional processes in ADHD and normal children Development of attentional processes in ADHD and normal children. Progress in Brain Research, 176, $259-76$.

Gupta, R. \& Kar, B. R. (2012). Attention and memory biases as stable abnormalities among currently depressed and currently remitted individuals with Unipolar Depression. Frontiers in Psychiatry, 3:99.

Gupta, R., Kar, B. R., \& Srinivasan, N. (2011). Cognitive-motivational deficits In ADHD: Development of a classification system. Child Neuropsychology, 17, 67-81.

Gupta, R., \& Raymond, J. E. (2012). Emotional distraction unbalances visual processing. Psychonomic Bulletin and Review, 19, 184-189.

Gupta, R., Raymond, J. E., \& Vuilleumier, P. (2019). Priming by motivationally salient distractors produces hemispheric asymmetries in visual processing. Psychological Research, 83, 1798-1807.

Gupta, R., \& Srinivasan, N. (2015). Only irrelevant sad but not happy faces are inhibited under high perceptual load. Cognition \& Emotion, 29(4), 747-754.

Hammer, J. L., \& Marsh, A. A. (2015). Why do fearful facial expressions elicit behavioral approach? Evidence from a combined approachavoidance implicit association test. Emotion, 15(2), 223-231.

Jackson, M. C., Wolf, C., Johnston, S. J., Raymond, J. E., \& Linden, D. E. J. (2008). Neural correlates of enhanced visual short-term memory for angry faces: an fMRI study. PLoS One, 3, e3536.

Kalanthroff, E., Cohen, N., \& Henik, A. (2013). Stop feeling: inhibition of emotional interference following stop-signal trials. Frontiers in Human Neuroscience, 7, 78. 
Kesler-West, M.L., Andersen, A.H., Smith, C.D., Avison, M.J., Davis, C.E., et al. (2001). Neural substrates of facial emotion processing using fMRI. Cognitive Brain Research, 11, 213-226.

Kleiner, M., Brainard, D. H., Pelli, D., Ingling, A., Murray, R., \& Broussard, C. (2007). What's new in Psychtoolbox-3. Perception, 36(14), 1.1-16.

Lang, P. J., \& Bradley, M. M. (2013). Appetitive and Defensive Motivation: Goal-Directed or Goal-Determined?. Emotion review: journal of the International Society for Research on Emotion, 5(3), 230-234.

Lang, P. J., Davis, M., \& Ohman, A. (2000). Fear and Anxiety: Animal Models and Human Cognitive Psychophysiology. Journal of Affective Disorders, 61, 137-159.

Lavie, N. (2005). Distracted and confused?: Selective attention under load. Trends in Cognitive Sciences, 9, 75-82

Leppänen, J. M., \& Hietanen, J. K. (2004). Positive facial expressions are recognized faster than negative facial expressions, but why? Psychological Research, 69, 22-29.

Logan, G. D., \& Cowan, W. B. (1984). On the ability to inhibit thought and action: A theory of an act of control. Psychological Review, 91(3), 295-327.

Logan, G. D., Schachar, R. J., \& Tannock, R. (1997). Impulsivity and inhibitory control. Psychological Science, 8(1), 60-64.

Lohani, M., Gupta, R., \& Srinivasan, N. (2013). Cross-cultural evaluation of the international affective picture system on an Indian sample. Psychological Studies, 58, 233-241.

Lundqvist, D., Flykt, A., \& Öhman, A. (1998). The Karolinska directed motional faces-KDEF CD-ROM. Stockholm: Department of Clinical Neuroscience, Psychology section, Karolinska Institutet.

Mack, A., \& Rock, I. (1998). MIT Press/Bradford Books series in cognitive psychology. Inattentional blindness. The MIT Press.

Marsh, A.A., Ambady, N., \& Kleck R.E., (2005). The effect of fear and anger facial expression on approach-and-avoidance-related behaviours. Emotion, 5(1), 119-24.

Marsh, A.A., Yu, H.H., Pine, D.S., \& Blair, R.J. (2010). Oxytocin improves specific recognition of positive facial expressions. Psychopharmacology, 209(3), 225-232.

Most S. B., Smith S. D., Cooter A. B., Levy B. N., \& Zald D. H. (2007). The naked truth: positive, arousing distractors impair rapid target perception. Cognition and Emotion, 21(5), 964-981.

Mostofsky, S.H., \& Simmonds, D.J. (2008). Response inhibition and response selection: two sides of the same coin. Journal of Cognitive Neuroscience, 20(5), 751-761.

Nikitin, J., \& Freund, A. M. (2019). Who cares? Effects of social approach and avoidance motivation on responsiveness to others. Personality and Social Psychology Bulletin, 45(2), 182-195.

Pandey, S., \& Gupta, R. (2019). Book Review: The influential mind: What the brain reveals about our power to change others. Frontiers in Psychology, 10, 1210.

Paulus, A., \& Wentura, D. (2016). It depends: Approach and avoidance reactions to emotional expressions are influenced by the contrast emotions presented in the task. Journal of Experimental Psychology: Human Perception and Performance, 42(2), 197-212.

Pawliczek, C. M., Derntl, B., Kellermann, T., Kohn, N., Gur, R. C., \& Habel, U. (2013). Inhibitory control and trait aggression: Neural and behavioral insights using the emotional stop signal task. Neuroimage, 79, 264-274.

Pessoa, L. (2009). How do emotion and motivation direct executive control?. Trends in cognitive Sciences, 13(4), 160-166.
Pessoa, L., McKenna, M., Gutierrez, E., \& Ungerleider, L. G. (2002). Neural processing of emotional faces requires attention. Proceedings of the National Academy of Sciences of the United States of America, 99(17), 11458-11463.

Pessoa, L., Padmala, S., Kenzer, A., \& Bauer, A. (2012). Interactions between cognition and emotion during response inhibition. Emotion, 12(1), 192-197.

Sagaspe P, Schwartz S, \& Vuilleumier P. (2011). Fear and stop: a role for the amygdala in motor inhibition by emotional signals. Neuroimage, 55(4), 1825-1835.

Savage, R. A., Becker, S. I., \& Lipp, O. V. (2016). Visual search for emotional expressions: Effect of stimulus set on anger and happiness superiority. Cognition and Emotion, 30, 713-730.

Scalzo, F., O’Connor, D. A., Orr, C., Murphy, K., \& Hester, R. (2016). Attention Diversion Improves Response Inhibition of Immediate Reward, But Only When it Is Beneficial: An fMRI Study. Frontiers in Human Neuroscience, 10: 429.

Sippel, L. M., \& Marshall, A. D. (2013). Posttraumatic Stress Disorder and Fear of Emotions: The Role of Attentional Control. Journal of Traumatic Stress, 26(3), 397-400.

Sprengelmeyer, R., Rausch, M., Eysel, U.T., \& Przuntek, H. (1998). Neural structures associated with recognition of facial expressions of basic emotions. Proceedings of Royal Society London B. 265, 1927-1931.

Srinivasan, N. \& Gupta, R. (2010). Emotion-Attention Interactions in Recognition Memory for Distractor Faces. Emotion, 10(2), 207-215.

Srinivasan, N., \& Gupta, R. (2011). Global-local processing affects recognition of distractor emotional faces. Quarterly Journal of Experimental Psychology, 64, 425-433.

Tian, Y., Liang, S., \& Yao, D. (2014). Attentional orienting and response inhibition: insights from spatial-temporal neuroimaging. Neuroscience Bulletin, 30, 141-152.

Tottenham, N., Tanaka, J. W., Leon, A. C., McCarry, T., Nurse, M., Hare, T. A., ... Nelson, C. (2009). The NimStim set of facial expressions: judgments from untrained research participants. Psychiatry Research, 168(3), 242-249.

Verbruggen F, Liefooghe B, \& Vandierendonck A. (2004). The interaction between stop signal inhibition and distractor interference in the flanker and Stroop task. Acta Psychologica, 116(1), 21-37.

Verbruggen, F., \& Logan, G. D. (2009). Models of response inhibition in the stop-signal and stop-change paradigms. Neuroscience and Biobehavioral Reviews, 33(5), 647-661.

Verbruggen, Frederick, \& De Houwer, J. (2007). Do emotional stimuli interfere with response inhibition? Evidence from the stop signal paradigm. Cognition and Emotion, 21(2), 391-403.

Vuilleumier, P. (2005). How brain beware: neural mechanism of emotional attention. Trends in Cognitive Sciences, 9(12), 585-594.

Link to download data: https://drive.google.com/file/d/ 1F0BVDXuv6w3X9i4hUn9cLdxBVov5oSTG/view?usp=sharing

Publisher's note Springer Nature remains neutral with regard to jurisdictional claims in published maps and institutional affiliations. 